\title{
Aproximación a los textos técnicos del Parlamento Europeo: los informes
}

(An approach to European Parliament technical texts: the case of reports)

\author{
María AZAHARA Veroz GonZÁleZ
}

(Grupo de Investigación HUM887)

Fecha de recepción: 23 de febrero de 2010

Fecha de aceptación: 15 de abril de 2010

Resumen: El presente trabajo se centra en identificar los rasgos lingüísticos característicos de un informe en su versión inglesa y su traducción paralela al español, pertenecientes a los textos técnicos emanados del Parlamento Europeo. Para identificarlos se analizarán los siguientes aspectos:

a) discursivos: gramática y léxico utilizados

b) lingüísticos y terminología jurídica,

c) retóricos: naturaleza de los textos y su finalidad.

Palabras clave: Traducción comunitaria, Parlamento Europeo, textos técnicos, informes, análisis lingüístico-discursivo.

Abstract:. This paper focuses on the identification of the linguistic features of a report in its English version and in its parallel Spanish translation, belonging to the technical texts emanated from the European Parliament. In order to identify these features the following aspects will be analysed:

a) discursive aspects: grammar and lexicon,

b) linguistic aspects and legal terminology,

c) rethorical aspects: text nature and purpose.

Key words: UE translation, European Parliament, technical texts, reports, linguistic discourse analysis.

INTRODUCCIÓN

Muchos son los enfoques de los que se han tratado de analizar los textos comunitarios Baker (1998) o Koskinen (2002) son algunos de ellos, aunque pocos se han centrado en el análisis de los textos de técnicos.

Según Garrido (2004) los textos técnicos son aquellos son todos aquellos que contienen datos, opiniones, juicios o análisis y que, en general, sirven de base para la preparación y adopción de los textos dispositivos o normativos. Dichos textos no tienen trascendencia jurídica, son de carácter informativo y no generan ni derechos ni obligaciones. 
Al realizar una revisión previa de los textos emanados de la Unión Europea, concretamente en el Parlamento Europeo, nos damos cuenta de que el grueso de la producción se encuentra en los textos técnicos y de carácter administrativo, y no en los textos dispositivos, los cuales tradicionalmente han sido analizados para determinar las características de la traducción comunitaria. Así pues, hemos creído necesario realizar un acercamiento a este tipo de textos.

En el estudio que ahora presentamos nos centraremos en identificar exclusivamente los rasgos lingüísticos característicos de un informe, pertenecientes a los textos técnicos anteriormente definidos.

Los pasos conducentes a tal estudio serán los siguientes:

a) identificar aspectos discursivos: gramática y léxico utilizados

b) identificar aspectos lingüísticos y terminología jurídica,

c) identificar aspectos retóricos: naturaleza de los textos y su finalidad.

Creemos que esta división es esencial puesto que la operación traductora está sometida a todas estas variables y es claramente dependiente de ellas. En efecto, con este tipo de textos el simple conocimiento lingüístico y el dominio de las palabras no son suficientes, sino que es necesario conocer todo lo que contextualmente rodea al documento y su realidad: una realidad definida a partir del ordenamiento jurídico.

Para investigar los aspectos discursivos de los textos nos hemos centrado principalmente en los estudios realizados sobre pragmática de los actos de habla y la cortesía en el estudio del discurso legal e institucional de diversos autores y en la teoría de los actos de habla de John L. Austin (1962) y John R. Searle (1976).

\section{LOS INFORMES}

Son el núcleo principal de la actividad parlamentaria. Normalmente, cuando un asunto pertenece al ámbito de competencia de una comisión determinada, se pide a ésta que elabore un informe; sin embargo, en determinadas circunstancias una comisión puede elaborar un informe por iniciativa propia. Los elabora un ponente (rapporteur, Berichtersttater) en nombre de una comisión parlamentaria y reciben enmiendas en comisión y en el Pleno.

Las ponencias se reparten entre los grupos políticos de acuerdo con un sistema de puntos. Existen muchos tipos diferentes de informe, pero básicamente se dividen en legislativos y no legislativos. Hasta su aprobación final en el Pleno un informe atraviesa, en general, las siguientes fases: documento de trabajo (DT), proyecto de informe (PR), enmiendas en comisión (AM), informe definitivo ( $R R$ - documento de sesión de tipo A7) y enmiendas en el Pleno (AM). El Servicio del Acta se encarga de incorporar 
al informe definitivo (RR/A7) las enmiendas aprobadas en el Pleno y de cotejar las distintas versiones lingüísticas. La propuesta legislativa (con o sin enmiendas) acompañada del proyecto de resolución legislativa o la propuesta de resolución pasan a formar parte del acta de la sesión y posteriormente se publican en el Diario Oficial. Cuando se cita un informe ya aprobado en el Pleno, se debe consultar la versión publicada en el acta, particularmente en el caso de informes en segunda y tercera lectura.

La estructura de un informe viene dada por la plantilla DocEp ${ }^{1}$, por lo que para el traductor, una vez que dispone de ella y sabe utilizarla, no supondrá ningún problema. No obstante, como aquí no disponemos de tal plantilla, hemos creído necesario explicar las partes de las que consta ya que han sido de mucha utilidad para el análisis posterior realizado:

- Una portada, en la que cabe destacar:

- En la primera línea del encabezamiento aparece el logotipo del Parlamento Europeo a la izquierda y a la derecha los años de la legislatura correspondiente. sesión".

En la última línea del encabezamiento aparece "Documento de

- Fecha: la fecha se escribe en cifras, sin ceros a la izquierda y con arreglo al formato "d.m.aaaa" en todas las lenguas (ejemplo: 2.5.2010)

- Identificación, a la derecha A7-9999/01 (A por "informe", 1 cifra para la legislatura $[A 7=2009-2014 ; A 6=2004-2009 ; A 5=1999-2004 ; A 4=$ 1994-1999], guión, cuatro cifras para un número secuencial, barra oblicua y dos cifras que indican el año) y el número PE del proyecto de informe.

- El título: tras el título que figura en la portada de un documento legislativo se consignarán, entre paréntesis la(s) referencia(s) $\mathrm{COM}^{2} \mathrm{o}$ el número del Consejo, el número de $\mathrm{C}$ (legislatura) $)^{3}$ y la signatura interinstitucional $^{4}$ en la que se señala el tipo de procedimiento, según sea un informe legislativo o no legislativo.

- El pie de página, en donde aparecerá, por un lado el número de hoja de transmisión ( $\mathrm{FdR}$ ) precedido de RR, por otro el número de

\footnotetext{
${ }^{1}$ Véase: "Cartilla práctica de DocEP". En: La Plataforma Internet de la Traducción Española en el Parlamento Europeo.

<http://www.europarl.europa.eu/transl_es/plataforma/pagina/hrdocep/hrdocepinstr00.htm>

[Consulta: 24/01/2010]

2 El número COM designa un documento de la Comisión.

${ }^{3}$ La referencia C(legislatura) se atribuye a un documento procedente de otra institución y anunciado en el Pleno.

${ }^{4}$ Véase: "Signaturas". En: La Plataforma Internet de la Traducción Española en el Parlamento Europeo

<http://www.europarl.europa.eu/transl_es/plataforma/pagina/guia/signaturas.htm> [Consulta: 20/01/2010]
} 
documento del Parlamento Europeo, y debajo de ambos el lema de la Unión Europea "Unida en la diversidad".

- Una página con informaciones prácticas, en el caso en que el informe sea legislativo, con dos cuadros: uno con la explicación de los signos utilizados y otro las normas de presentación de las enmiendas.

- Un índice: todas las partes del índice aparecen en mayúsculas y los nombres de las comisiones parlamentarias aparecerán en su versión completa.

- Una página en blanco, sólo para el caso de los informes legislativos, con la finalidad de que el informe comience en página impar.

- Las páginas que examina el Pleno: proyecto de resolución legislativa, posibles propuestas de enmiendas y justificaciones a las enmiendas.

- El proyecto de resolución legislativa se compone de guiones. El primer guión remite al texto al que se refiere el proyecto, éste irá seguido de otros guiones que hacen referencia a posiciones anteriores referentes al mismo procedimiento mencionado en el primer guión. A continuación van los guiones relativos a los fundamentos jurídicos comenzando por el artículo en virtud del cual el Consejo consulta al Parlamento o la Comisión le presenta la propuesta (en el proceso de codecisión, hoy llamado procedimiento legislativo ordinario ${ }^{5}$ ). Tras éste irá otro guión en el que figura el fundamento reglamentario por orden de importancia y el último guión se referirá siempre al informe o a la recomendación de la comisión competente y a las opiniones adjuntas si las hubiere.

- Enmiendas: Suelen ir en columnas y pueden ir acompañadas de una justificación sucinta.

- Exposición de motivos, es facultativa y puede incluir una ficha de financiación.

- Si existe una opinión minoritaria: se incluyen en el documento de sesión.

- En su caso, las opiniones de otras comisiones: se incluyen en el documento de sesión.

- Si procede la declaración de la Comisión sobre las enmiendas del Parlamento Europeo.

\footnotetext{
${ }^{5}$ El Procedimiento Legislativo ordinario (PLo), antes del Tratado de Lisboa conocido como el procedimiento de codecisión, es el principal proceso legislativo por el que un proyecto legislativo puede ser aprobado en la Unión Europea, exceptuándose algunas áreas sometidas a procedimiento legislativo especial (PLe) (sólo del Consejo o del Parlamento Europeo, aisladamente) y las que se excluyen de la legislación, como la Política Exterior y de Seguridad Común.

EI PLo atribuye al Parlamento Europeo y al Consejo el poder legislativo de la Unión, previa propuesta de ley presentada por la Comisión Europea, en igualdad de condiciones, para lo que se requiere la aprobación de un mismo texto por parte de las dos Instituciones, como cámaras legislativas colegiadas, en un sistema de bicameralismo perfecto.
} 
- Una página de procedimiento en forma de cuadro en el informe legislativo, en donde aparecerá un cuadro que contendrá las rúbricas pertinentes para el informe y reflejará el desarrollo del procedimiento hasta llegar a la fase de aprobación del informe. En el caso de un informe no legislativo aparecerá una página con resultado de la votación en forma de cuadro.

Para cada informe se elige, de entre los miembros de la comisión, a un ponente que se encargará de elaborarlo. Una vez redactado, el proyecto de informe se presenta y examina en reuniones de comisión; los diputados pueden proponer enmiendas al texto, si lo desean. Los proyectos de informe son aprobados en comisión parlamentaria y presentados para su aprobación en sesión plenaria. Después de aprobados por la comisión parlamentaria competente para el fondo y después de presentados al servicio de la Sesión, los informes son examinados en sesión plenaria.

\section{ANÁLISIS LINGÜÍSTICO-DISCURSIVO}

El estudio lingüístico de los textos técnicos y administrativos del Parlamento Europeo ha de realizarse en el marco de los métodos propuestos por la disciplina de Análisis del Discurso, rama que se ha desarrollado en los últimos años y se ha aplicado en muchas direcciones en las que la contextualización del uso de la lengua tiene un carácter hasta cierto punto diferente, especial o no previsto en el uso cotidiano.

El objetivo principal de tal estudio ha sido tal y como hemos explicado anteriormente identificar los rasgos lingüísticos característicos de los textos técnicos y de comunicación administrativa del Parlamento Europeo a través de los siguientes pasos:

a) identificación de los aspectos discursivos: gramática y léxico utilizados

b) identificación de los aspectos lingüísticos y terminología jurídica e institucional,

c) identificación de los aspectos retóricos: naturaleza de los textos y su finalidad.

Efectivamente, no basta con simplemente conocer este tipo de textos, pues el conocimiento lingüístico y el dominio terminológico no son suficientes, sino que estimamos necesario llegar a tener un cabal conocimiento de lo que contextualmente rodea al documento y su realidad: una realidad definida a partir del ordenamiento jurídico.

2.1. Identificación de rasgos discursivos de los textos: gramática y léxico utilizados, e identificación de los aspectos retóricos: naturaleza de los textos y su finalidad

Este tipo de dominio que participa del lenguaje y jerga legal y comunitaria (eurospeak) dentro del medio discursivo escrito es "único" en lo 
que concierne a sus rasgos léxicos, como términos técnicos, expresiones arcaicas, etc., además de ser conocido por su sintaxis a menudo compleja, que ha merecido estudios específicos (van Dijk, 1985). No obstante, a excepción de algunos estudios (Yon Maley, 1987, Werther and Herlmersen, 1989, Myers 1989 y Pilegaard 1990, Blom y Trosborg, 1992,) se ha prestado poca atención a los estudios discursivos (pragmática de los actos de habla y la cortesía) en el estudio del discurso legal e institucional.

Los rasgos que tradicionalmente se asignan al lenguaje legislativo e institucional es el de arcaico y extraño en contraste con el lenguaje cotidiano, casual o familiar. Al mismo tiempo en sintaxis tendríamos frases complejas y largas, bien sean coordinadas o subordinadas (Crystal and Davy, 1969; Bhatia, 1982) uso predominante de la voz pasiva (Danet, 1980; 1985) y ausencia de agentes humanos (Finegan, 1982).

El lenguaje, esencial en los asuntos humanos, asume un papel particularmente crítico en lo que a legislación concierne. De hecho, en un sentido muy elemental, la ley no existiría sin el lenguaje. Cabe distinguir dos funciones primarias de la ley: a) la ordenación de las relaciones humanas y b) la restauración del orden social cuando éste se quiebra. Con referencia a la primera función la ley tiene dos vertientes: la regulativa y la constitutiva. Por ejemplo, la denominada "enacting formula" británica o también llamada en español de "promulgación" tiene estos ingredientes lingüísticos básicos:

\section{BE IT ENACTED by the Queen....}

Es decir, la correcta formulación es garantía del éxito de la promulgación. De hecho, este rasgo determina que el acto en cuestión sea, desde el punto de vista discursivo, una "declaración", que es la conditio sine qua non sobre la que está efectuado su estatuto de ley. Es decir, formulada de otra manera no tendría efecto pretendido.

De la misma manera, los documentos que aquí examinamos, al igual que otro tipo textos legales o contratos, deben estar escritos con las fórmulas lingüísticas apropiadas y quedar debidamente firmados para ser válidos legalmente. El texto legal británico, por ejemplo, es un acto de habla performativo que contiene el verbo preformativo to enact que establece la fuerza elocutiva de todo el texto, esto es, su macro-función. Por otra parte, el otro ejemplo, la comunicación contractual, es única en que las intenciones relativas de las partes se expresan por escrito con la ayuda de un filtro intermediario -alguien versado en leyes- con referencia especial a las limitaciones legislativas -la base del contrato- y con vistas a la institución que dirime la disputa, el tribunal de justicia. La distribución de los roles es fija (la asignación de roles es convencional (comprador / vendedor, etc.) y la relación entre ellos es en principio simétrica, pues ambos ofrecen algo 
(promesa - consideración) que contrasta con la relación asimétrica existente entre el poder legislativo y los ciudadanos. El primero promulga leyes para ser obedecidas por los ciudadanos con objeto de mantener la ley y el orden.

En nuestro caso concreto, los informes, estamos hablando de textos técnicos y comunicaciones administrativas, producidos en el seno del Parlamento Europeo. Estos textos pueden ser, como ya hemos explicado con anterioridad, legislativos o no, pudiendo pertenecer al procedimiento legislativo ordinario (hasta hace poco denominado procedimiento de codecisión) por el cual un proyecto legislativo puede ser aprobado en la Unión Europea, exceptuándose algunas áreas sometidas a procedimiento legislativo especial (PLe), por el Parlamento Europeo y el Consejo, previa propuesta de la Comisión Europea en igualdad de condiciones.

Así pues, en el informe analizado en inglés tenemos:

DRAFT EUROPEAN PARLIAMENT LEGISLATIVE RESOLUTION

AIM: adopting a regulation of the European Parliament and of the Council establishing a European Asylum Support Office.

Y su traducción al español se formula así: EUROPEO

PROYECTO DE RESOLUCIÓN LEGISLATIVA DEL PARLAMENTO

OBJETIVO: con vistas a la adopción del Reglamento del Parlamento Europeo y del Consejo por el que se crea una Oficina Europea de Apoyo al Asilo.

La recomendación de una segunda lectura con vistas a una resolución viene formulada de la siguiente forma:

The European Parliament,

1. Approves the Council's position;

2. Notes that the act is adopted in accordance with the position;

3. Instructs its President to sign... así:

En español, el proyecto de esta resolución está, a su vez, formulada

El Parlamento Europeo:

1. Aprueba la Posición del Consejo;

2. Constata que el acto ha sido adoptado con arreglo a la Posición;

3. Encarga a su Presidente que firme el acto.

Luego, los tres actos básicos de discurso ilocutivo en el primer texto se cimentan en estos tres fundamentales: en inglés: approves, notes e instructs que se traducen por: aprueba, constata y encarga.

La traducción es, pues, única en el sentido de que no sería lo esperable en otro tipo de discurso y en otro contexto donde los verbos utilizados en dichos textos podrían tener otros usos muy distintos. Son, 
pues, fórmulas a las que han llegado los traductores utilizando verbos que son, en cierto sentido, polisémicos.

En este análisis, la autoridad de donde dimana el poder es: el Parlamento Europeo, y no el rey o la reina como suele ocurrir en las monarquías con respecto a las leyes o el Gobierno en los decretos-leyes.

Cabe destacar otra característica propia tanto del discurso legal como del discurso institucional, el empleo abundante de siglas y acrónimos para referirse a instituciones, programas, organismos y conceptos en ambos idiomas y en los tres textos que hemos analizado, así pues obtenemos:

\begin{tabular}{|c|c|}
\hline INGLÉS & ESPAÑOL \\
\hline EC & CE \\
\hline ERF & FER \\
\hline LIBE & LIBE \\
\hline COREPER & COREPER \\
\hline UNHCR & ACNUR \\
\hline
\end{tabular}

Así mismo, hemos de destacar la repetición de palabras con la finalidad de ser objetivo en el discurso no admitiendo palabras polisémicas dentro del mismo discurso, así encontramos: Conseil (Consejo) para referirse al Consejo Europeo, Commission (Comisión) para la Comisión Europea, Estados miembros o Fondo.

También nos hemos encontrado con palabras cuyo uso es compartido por varios tecnolectos o el lenguaje común pero que son tomadas en acepciones diferentes y propias del lenguaje jurídico tales como: article (artículo), fund (fondo), program (programa) o report (informe).

\subsection{La función comunicativa: los actos regulativos}

El lenguaje es crítico al regular la conducta social humana. Con el fin específico de construir estatutos u otro tipo de reglas el lenguaje "organiza y regula las relaciones humanas" es decir, tiene una función regulativa.

En la taxonomía establecida por John R. Searle, ya en 1976, en "Speech Acts" establece dos grandes categorías de actos regulativos: directives y commisives, cuya razón de ser estriba en la intención del "hablante" en regular el "mundo" con las palabras, opuesto, en cierto sentido, por ejemplo, a los actos representativos en los que las palabras se ajustan para adaptarse al mundo, según la explicación de J. R. Searle. 
Los principales actos representativos que resumen lo propuesto por Searle podrían ser los de: request y promise, por más que difieran en su estudio ambas categorías pragmáticas.

Mientras que el poder legislativo es claramente coercitivo, los compromisos adquiridos en un contrato, por ejemplo, pueden establecerse como una obligación emitida por una de las partes sobre la otra o por una parte que se compromete a sí misma, o parte de ella. En el caso del Parlamento Europeo, las comisiones elaboran un informe sobre asuntos legislativos o no legislativos, una vez examinados por la comisión encargada, dichos informes se presentarán en Pleno para ser votados. Así pues, en el texto estudiado el Parlamento Europeo aprueba, bilateralmente junto con el Consejo, la propuesta de reglamento realizada por la Comisión al Parlamento y al Consejo, para establecer una oficina de apoyo al asilo.

De acuerdo con Austin en su obra pionera sobre los "Actos de habla" (Speech acts), titulada "How to do things with words", publicada en 1962, "the whole point of a commissive is to commit the speaker to a certain course of action". Ejemplos de ello son verbos del tipo: promise, vow, pledge, contract, guarantee, embrace, swear, etc., entre otros. Searle añadió algunas objeciones a Austin, ya que, a su parecer, algunos de esos verbos no pertenecían a la categoría antedicha.

Así que Searle señala: "Commissives then are those illocutionary acts whose point is to commit the speaker (again in varying degrees) to some future course of action". Establece algunas condiciones, como la de "sinceridad": la intención misma. El compromiso de la acción a acometer (comisiva) es expresada con un verbo performativo explicito: prometo, etc. o por medio de un verbo modal en inglés o el tiempo verbal de futuro en español. Las promesas van en beneficio de los oyentes o audiencia (aquí los ciudadanos) y G. Leech (1983) las incluye en la categoría pragmática de "convivial acts". Está claro que los actos comisivos legislativos (emanados de organismos de forma corporativa) son parecidos pero algo distintos a los meramente realizados entre seres humanos particulares.

DATA: los data de la investigación se han extractado del corpus de lenguaje institucional dentro del campo específico de:

A. PROYECTO DE RESOLUCIÓN LEGISLATIVA DEL PARLAMENTO EUROPEO sobre la Posición del Consejo en primera lectura con vistas a la adopción del Reglamento del Parlamento Europeo y del Consejo por el que se crea una Oficina Europea de Apoyo al Asilo.

En inglés el primer texto resulta de la siguiente manera:

\begin{tabular}{|l|c|}
\hline Antecedents (8 acts) & Having regard to... \\
\hline Aproval (1 act) & Approves the Council's \\
position
\end{tabular}




\begin{tabular}{|l|l|}
\hline Notice (1 act) & $\begin{array}{c}\text { Notes that the act is adopted } \\
\text { in accordance with the position; }\end{array}$ \\
\hline Instruction (1 acts) & Instructs its President to \\
\hline
\end{tabular}

Tabla 2.Estructura de la Propuesta legislativa en inglés

En español:

\begin{tabular}{|c|l|}
\hline Antecedentes (8 actos) & $\begin{array}{c}\text { Vista la posición, vista la } \\
\text { propuesta, vistos los artículos, vista } \\
\text { la recomendación... }\end{array}$ \\
\hline Aprobación (1 acto) & $\begin{array}{c}\text { Aprueba la posición del } \\
\text { Consejo }\end{array}$ \\
\hline Constatación (1 acto) & $\begin{array}{l}\text { Constata que el acto ha sido } \\
\text { adoptado según la antedicha } \\
\text { posición. }\end{array}$ \\
\hline Encargo (1 acto) & $\begin{array}{c}\text { Encarga a su presidente } \\
\text { que...(2), a su Secretario que...(1) }\end{array}$ \\
\hline
\end{tabular}

Tabla 3. Estructura de la Propuesta legislativa en español

El preámbulo de la exposición es una preliminar explicación de los motivos de la declaración. Hay dos proposiciones diferentes: la primera es una narración (la Comisión adoptó...la propuesta de Reglamento...por la que se crea una Oficina...con una especificación de la fecha de la medida tomada (18 de febrero). La segunda es la exposición de los objetivos de la susodicha Oficina, que es prestar asistencia en orden a contribuir a alcanzar una política coherente y de gran calidad en el asunto de Asilo.

\section{EXPLANATORY STATEMENT}

The Commission adopted on 18 February 2009 the proposal for a regulation establishing a European Asylum Support Office (Support Office) and the accompanying proposal amending the Decision on the European Refugee Fund (ERF). The aim of the Support Office is to provide the necessary expert assistance to help in the delivery of a consistent and high quality Common European Asylum Policy.

\section{EXPOSICIÓN DE MOTIVOS}

La Comisión adoptó el 18 de febrero de 2009 la propuesta de Reglamento del Parlamento Europeo y del Consejo por el que se crea una Oficina Europea de Apoyo al Asilo (Oficina de Apoyo) y la propuesta de acompañamiento para modificar la Decisión sobre el Fondo Europeo para los Refugiados (FER). El objetivo de la creación de la Oficina de Apoyo es prestar la asistencia especializada 
necesaria para contribuir a alcanzar una Política Europea Común de Asilo coherente y de alta calidad.

La exposición de motivos consta de dos partes muy distintas:

a) Procedimientos (procedure): una cronología de los hechos que desencadenaron el proyecto de la resolución parlamentaria, relatados en tiempo de pretérito (past tense) sobre los pasos que se iban dando hacia esa resolución.

b) El contenido (content): es una exposición de actos comisivos redactados en tiempo verbal de futuro o modal comisivo (will) en inglés.

El primero está organizado de la siguiente manera:

(a) El 7 de mayo de 2009, el Parlamento Europeo aprobó su posición en primera lectura sobre la Oficina de Apoyo y la modificación del FER.

(b) Tras las elecciones, se reanudó el procedimiento con el nuevo Parlamento. Se confirmó a Jean Lambert como ponente y [...]

(c) La Comisión de Libertades Civiles, Justicia y Asuntos de Interior (LIBE) le encomendó que negociara el texto con el Consejo y la Comisión (30 de septiembre).

(d) Las negociaciones tuvieron lugar bajo la Presidencia sueca, desde finales de septiembre hasta principios de noviembre.

(e) Se celebraron cuatro diálogos a tres bandas (1 de octubre, 8 de octubre y 5 de noviembre).

(f) El ponente y los ponentes alternativos colaboraron estrechamente $e$ informaron regularmente a la Comisión LIBE.

(g) El 12 de noviembre tuvo lugar una votación orientativa sobre los textos de transacción, que reflejaban el compromiso alcanzado por el ponente, los ponentes alternativos, la Comisión y el Consejo.

(h) El Presidente de la Comisión LIBE, Juan Fernando López Aguilar remitió una carta al Consejo.

(i) El 18 de noviembre se alcanzó un acuerdo sobre ambos textos en el COREPER.

(j) A continuación, los juristas lingüistas de ambas instituciones examinaron los textos, que fueron aprobados por el Consejo los días 25 y 26 de febrero.

(k) Las dos posiciones del Consejo se remitieron al Parlamento Europeo y se anunciaron en el Pleno el 10 de marzo de 2010. 
(I) El 16 de marzo de 2010 se presentaron en la Comisión LIBE los proyectos de recomendación y la votación tendrá lugar los días 7 y 8 de abril.

La exposición paralela en inglés se ciñe estrictamente a la española que hemos visto: verbos de acción con sujetos distintos, sobre todo no de persona (excepto el colectivo Comisión). En inglés la voz pasiva surge de la consabida nominalización en la que el sujeto es un nombre abstracto y no personal.

Veamos, pues, la parte inglesa:

(a) On 7 May 2009, the European Parliament adopted its first reading position on the Support Office and the change to the ERF.

(b) After the elections, with the newly elected Parliament, the procedure continued.

(c) Jean Lambert was confirmed as rapporteur and was given the mandate by the Committee on Civil Liberties, Justice and Home Affairs (LIBE) to negotiate with Council and Commission on the text (on 30 September).

(d) The negotiations took place under the Swedish Presidency, from the end of September until early November.

(e) Four trilogues took place (1 October, 8 October, 22 October and 5 November).

(f) The rapporteur and the shadow rapporteurs worked closely together and regular feedback was given to the LIBE committee.

(g) An orientation vote on the compromise texts, which reflected the compromise between the rapporteur, the shadow rapporteurs, Commission and Council, was held on 12 November.

(h) The LIBE Chairman, Juan Fernando López Aguilar, sent a letter to the Council.

(i) On 18 November agreement was reached in COREPER on both texts.

(j) Then the lawyer-linguists of both institutions worked on the texts and on 25 and 26 February they were adopted by the Council.

(k) Both Council positions were sent to the European Parliament and announced in plenary on 10 March 2010.

(I) Draft recommendations were presented in LIBE on 16 March 2010 and a vote will take place on 7/8 April. 
Como decíamos el contenido (content) es una exposición de actos comisivos redactados en tiempo verbal de futuro en español y modal comisivo (will) en inglés.

(a) La Oficina de Apoyo será competente para coordinar los intercambios de información y otras acciones de reasentamiento adoptadas por los Estados miembros..

(b) Se prevé que el candidato escogido por el Consejo de Administración realizará una declaración ante la comisión o comisiones competentes del Parlamento Europeo.

(c) tras lo cual el Parlamento podrá aprobar un dictamen.

(d) El Consejo de Administración informará al Parlamento Europeo sobre la forma en que se ha tenido en cuenta dicho dictamen.

(e) La solidaridad intracomunitaria se basará en un acuerdo entre los Estados miembros

(f) El Estado miembro, cuando proceda, consultará con el Alto Comisionado de las Naciones Unidas

(g) A petición del Parlamento Europeo se incluye una disposición sobre el Foro consultivo, que constituirá un mecanismo para el intercambio de información...

(h) Garantizará un diálogo directo entre la Oficina de Apoyo y las demás partes interesadas.

(i) El Parlamento Europeo considera que los cambios presupuestarios necesarios en el Fondo Europeo para los Refugiados proporcionarán una financiación adecuada...

En inglés esta previsión de la propuesta que será aprobada:

(a) The Support Office will be responsible for coordinating exchanges of information and other actions

(b) It is foreseen that the candidate selected by the Management Board will make a statement

(c) after which the Parliament may adopt an opinion.

(d) The Management Board shall inform the European Parliament of the manner in which that opinion is taken into account.

(e) Intra community solidarity shall take place on an agreed basis between the Member States and with the consent of the individual concerned 
(f) The Member State may, where appropriate, consult the United Nations High Commissioner for Refugees

(g) On request of the European Parliament there is a provision on the Consultative Forum which shall constitute a mechanism for the exchange of information.

(h) It shall ensure that there is a close dialogue between the Support Office and the relevant stakeholders.

(i) The European Parliament considers that the necessary budgetary changes to the European Refugee Fund will provide adequate funding for the new agency in its initial phases.

En definitiva, donde el español expresa con tiempo de futuro las recomendaciones de la resolución legislativa, en inglés hay una matización de las acciones futuras, dependiendo de si significan modalidad epistémica o facultativa que propicia el uso de may (en los casos de c y f de la descripción del texto) y cuando es deóntica o de obligación (con uso de shall y de will). Ambos tipos de modalidad quedan expresados por el futuro en español, que es por tanto ambiguo (Givón 1989) en cuando a su especificación del sentido modal.

Con respecto a la utilización del shall nos gustaría hacer mención al artículo $n^{\circ} 3$ (“Una cultura jurídica común. ¿Ficción o realidad?") del Vademécum del traductor externo, escrito por Edward Seymour.

El uso de esta forma se remonta a los primeros textos escritos en inglés y ciertamente a su uso más famoso en la Biblia del Rey Jacobo de 1611 y, con anterioridad, a la traducción de los Diez Mandamientos que hizo William Tyndale en 1530. Los autores de textos jurídicos en inglés se han esforzado por utilizar shall de forma adecuada, confundidos no sólo por las dudas que se les planteaban a la hora de definir qué es un texto jurídico, sino también por las interferencias con la forma correcta del futuro en inglés: "I shall, you will, she will, we shall, you will, they will", sin olvidarse de la preferencia generalizada en el inglés vernáculo por las contracciones "I'll, you'll, they'll'.

En una conferencia sobre traducción jurídica celebrada en Ginebra en febrero de 2000, uno de los ponentes citó el siguiente mantra como recordatorio de la distinción entre shall con valor de futuro y shall en sentido jurídico: salvará)

"I shall drown, and no one will save me" (me ahogaré y nadie me

"I will drown, and no one shall save me" (es mi intención ahogarme, y nadie debe hacer nada para salvarme).

¿Cuál es la convención cultural equivalente en las demás lenguas de la UE? En la Guía Práctica Común se establece la norma: 
En la parte dispositiva de los actos de carácter vinculante, los verbos se emplearán en francés en presente de indicativo, mientras que la fórmula utilizada generalmente en inglés será "shall" seguido de infinitivo. En ambas lenguas debe evitarse el futuro en la medida de lo posible. En cambio, el uso del futuro normativo en castellano es preceptivo.

2.3. Identificación de los aspectos lingüísticos y terminología jurídica e institucional

\subsubsection{Fraseología}

Los presentes textos presentan una característica particular que es el empleo de locuciones preposicionales complejas, muchas con la estructura $\mathrm{P}+\mathrm{N}+\mathrm{P}$. El traductor debe saber cómo se pueden traducir para mantener el lenguaje jurídico. A veces hay más de una posibilidad de traducción, por ejemplo "pursuant to" puede ser "con arreglo a" o "en virtud de", lo importante es mantener el sentido.

Así pues, identificaremos algunas de las locuciones preposicionales más frecuentes en los informes estudiados, incluiremos el número de veces que aparecen, cómo se han traducido y además mostraremos un ejemplo:

a) Pursuant to (4)/ Conforme a/ De conformidad:

"Having regard to Article 251(2), Article 63, first paragraph, points (1) and (2), and Article 66 of the EC Treaty, pursuant to which the Commission submitted the proposal to Parliament (C6-0071/2009)".

Traducido por: "Vistos el artículo 251, apartado 2, el artículo 63, párrafo primero, puntos 1 y 2 , y el artículo 66 del Tratado CE, conforme a los cuales la Comisión le ha presentado su propuesta (C6-0071/2009)".

b) In accordance with (8) / Con arreglo a/ De acuerdo con/ De conformidad con/ Según:

"Notes that the act is adopted in accordance with the position".

"Constata que el acto ha sido adoptado con arreglo a la Posición".

c) In the appointment of (1) / en el nombramiento de:

"The role of the European Parliament in the appointment of the Executive Director was an important issue".

Y su traducción al español:

"Un punto importante fue la intervención del Parlamento Europeo en el nombramiento del Director Ejecutivo".

De la misma manera que encontramos este tipo de locuciones preposicionales que a menudo aparecen en el lenguaje jurídico, hemos de destacar también la aparición de colocaciones también propias del lenguaje jurídico en ambos idiomas tales como:

a) Having regard traducido como Visto/a.

Por ejemplo: 
"Having regard to the United Nations Millennium Declaration of 8 September 2000".

"Vista la Declaración del Milenio de las Naciones Unidas de 8 de septiembre de 2000".

b) Where appropriate traducido por Cuando proceda.

Por ejemplo:

"The Member State may, where appropriate, consult the United Nations High Commissioner for Refugees (UNHCR)".

"El Estado miembro, cuando proceda, consultará con el Alto Comisionado de las Naciones Unidas para los Refugiados (ACNUR)".

\subsection{Terminología}

Con respecto a la terminología encontrada en este informe conviene destacar la aparición del llamado eurospeak o jerga comunitaria, en términos como reglamento o directiva.

Enfatizaremos en este punto que el eurospeak puede presentar dos formas: una inevitable, correcta y nada controvertida y otra que se debe evitar.

1. Según esto se podría decir que, como el ordenamiento jurídico de la Unión Europea tiene un carácter genuino, es inevitable y normal que sus conceptos resulten más o menos extraños respecto a los ordenamientos jurídicos, por ello, en este caso se justifica el eurospeak.

2. Sin embargo, en la mayoría de los casos, los textos tratan sobre temas que también se abordan en ministerios, direcciones generales o en otros organismos a nivel nacional de acuerdo con convenciones de expresión naciones, en cuyo caso el llamado eurospeak no estaría justificado (Strandvik, 2002).

\section{CONCLUSIONES}

A lo largo de los puntos anteriores se ha desarrollado un modelo de análisis del discurso aplicado al informe estudiado, con el fin de definir de manera inicial las características propias de los informes y por consiguientes de los textos de carácter técnico emanados en el Parlamento Europeo.

A continuación presentamos una síntesis de las principales conclusiones y aportaciones de nuestro estudio,

Rasgos discursivos (gramática y léxico utilizados) y aspectos retóricos (naturaleza del texto y su finalidad)

El informe analizado se caracteriza principalmente por:

- Utilizar un lenguaje muy similar al lenguaje jurídico y administrativo. 
- Empleo abundante de siglas y acrónimos para referirse a instituciones, programas, organismos y conceptos.

- Repetición de palabras con la finalidad de ser objetivo en el discurso.

- Empleo de palabras compartido por varios tecnolectos o lenguaje común.

- Utilización de verbos de acción con diferentes sujetos, sobre todo no de persona (experto los colectivos: Comisión, Parlamento y Consejo).

- Empleo de la voz pasiva, que en inglés surge de la consabida nominalización en la que el sujeto es un nombre abstracto y no personal, mientras en español se opta por la pasiva refleja.

- Empleo excesivo de las formas no verbales del verbo, en concreto del infinitivo para expresar objetivos y el gerundio que el traductor en la mayoría de los casos traduce por un infinitivo.

De acuerdo con la estructura de los informes que constan de las siguientes partes básicas (se pueden añadir partes como hemos visto reflejado en el tercer texto traducido) y que por tanto caracterizan a dicha tipología de textos:

- Proyecto de resolución: caracterizado por unos antecedentes (antecedents), considerandos (accounts), aprobación (aproval), constatación (notice) y encargo (instruct).

- Exposición de motivos: dividida en tres partes principalmente:

- Contexto o antecedentes: cronología de los actos, redactados principalmente en tiempo de pretérito (past tense) o presente histórico (historical past).

- Constatación de los hechos en el momento actual: redactados principalmente en presente (present tense).

- Recomendaciones de propuesta legislativa: expresada en el tiempo futuro o modal comisivo (will, shall, may, must, should, need). Conviene señalar en este punto que donde el español se expresa con tiempo de futuro, en inglés hay una matización de las acciones futuras, dependiendo de si significan modalidad epistémica o facultativa que propicia el uso de may (en los casos de c y f de la descripción del texto) y cuando es deóntica o de obligación (con uso de shall y de will). Ambos tipos de modalidad quedan expresados por el futuro en español, que es por tanto ambiguo (Givón, 1989) en cuando a su especificación del sentido modal.

- Procedimiento: se trata de un cuadro con un resumen del procedimiento. 
Aspectos lingüísticos y terminología jurídica e institucional

Los textos técnicos y de comunicación administrativa, en concreto, los informes, se caracterizan por el empleo de locuciones preposicionales complejas, también características del lenguaje jurídico y administrativo.

La terminología empleada, si bien la mayoría corresponde al campo semántico del tema del informe en cuestión, convendría señalar que existe una terminología propia comunitaria, llamada por varios autores eurospeak. No se debe abusar de dicha terminología, es decir, se debe emplear únicamente cuando se hable del ordenamiento jurídico de la UE, ya que el empleo excesivo de esta terminología propia de la UE podría dificultar la aplicación y el traslado de algunas acciones o normativas a los sistemas legislativos nacionales.

REFERENCIAS BIBLIOGRAFICAS

AUSTIN, J.L.: How to do things with words. New York: Oxford U. Press. 1962.

BAKER, Mona Y SALDANHA, Gabriela: Routledge Encyclopedia of Translation Studies. Routledge: Abingdon, 1962, 2ª ed. 2009.

BHATIA, V. K.: "Classification of legislative texts" En: English for Specific Purposes, 68. Cornvallis: English Language Institute at Oregon State University. 1982. Pp. 8-9.

BIBER, Douglas: Variation across speech and writing. Cambridge U. Press. 1988.

BORJA ALBI, Anabel: Estrategias, materiales y recursos para la traducción jurídica inglés español. Madrid: Edelsa Grupo Didascalia, S.A.

COMUNIDADES EUROPEAS: Guía práctica común del Parlamento Europeo, del Consejo y de la Comisión [en línea]. Luxemburgo: Oficina de Publicaciones Oficiales de las Comunidades Europeas. 2003. [Consulta: 01/01/2010] Disponible en Internet:

$<$ http://eur-lex.europa.eu/es/techleg/pdf/es.pdf>

CRYSTAL, D. \& DAVY, D.: Investigating English Style. London: Longman. 1969.

CUNNIGHAM, K.: "Translating for a larger Union. Can we core with more than 11 languages?" [en línea]. En: Terminologie et Traduction, Bruselas. 2001. [Consulta: 16/01/2010]. Disponible en Internet:

<http://ec.europa.eu/translation/reading/articles/pdf/2001_cunningham.pdf>

DANET, Brenda: "Language in the legal process" En: Law and Society Review 14. 1982. Pp. 445-563

"Legal discourse", En: Teun van Dijk (ed) Handbook of Discourse Analysis, Vol I, London: Academic Press. 1985. Pp. 273-291. 
FERNÁNDEZ MIRANDA, Ma E. "La traducción en las instituciones europeas y en las organizaciones internacionales". Hieronymus Complutensis, ํo 4. 1996 - 1997. Pp. 87-100.

FUENTE, Félix De La: Diccionario jurídico de la Unión Europea. Barcelona: PPU. 1994.

GARCIA YeBRA, Valentín: Teoría y práctica de la traducción, 2 vols., Madrid, Gredos. 1982.

GARRIDO NOMBELA, Ramón: "La traducción en la Comunidad Europea y el lenguaje jurídico comunitario". Hieronymus Complutensis, n03. 1996. Pp. 35-41.

Y NAVARRETE, Miguel Á. "Traducción institucional: el caso de la Unión Europea”. En: CRUCES ColAdo, Susana Y LUNA ALONSO, Ana (eds.) La traducción en el ámbito institucional: autonómico, estatal y europeo. Vigo: Universidade de Vigo. 2004. Pp. 151-186.

GIL CATALINA, Gonzalo: La traducción institucional. La traducción en el PE, Notas, Luxemburgo, 2001.

GıvóN, Talmy: Mind, Code and Context. Hillsdale: Lawrence Erlbaum. 1989.

Grupo De Trabajo Sobre El MultilingüIsmo en la Perspectiva De La AMPLIACIÓN: Informe definitivo, PE, Luxemburgo, 1999.

HATIM, B., MASON, I. Discourse and the Translator. London: Longman. 1991.

KOSKINEN, Kaisa. "How to research EU Translation", En: PerspectivesStudies in Translatology, volumen 9:4. 2001. Pp. 293 - 300.

LABRIE, N. La construction linguistique de la Communauté européenne. 1:edición. París: Champion. 1993.

LEECH, Geoffrey: Principles of Pragmatics. London: Longman. 1983.

MALEY, Yon: "The language of legislation" En: Language in Society 16. 1987. Pp. 25-46

MARTín RuANO, $\mathrm{M}^{\mathrm{a}}$ del Rosario. "Teorías y utopías: hacia nuevos vocabularios y prácticas de la traducción institucional" [en línea]. En: Puntoycoma, Boletín de los traductores españoles de las instituciones de la Unión Europea. № 177. 2010. Disponible en Internet. [Consulta 26/01/2010] $<$ http://ec.europa.eu/translation/bulletins/puntoycoma/117/pyc11722_e s.htm>

MAYORAL, Roberto: "Las fidelidades del traductor jurado: batalla indecisa". Pp. 17 - 57. En: FERIA, Manuel (ed.). Traducir para la Justicia. Comares: Granada. 1999.

Newmark, Peter. Manual de traducción, Madrid, Cátedra. 1992.

MYERS, Greg: "The pragmatics of scientific articles" En: Applied Linguistics 10. 1989. Pp 1-35.

PARLAMENTO EUROPEO. "Vademécum del Traductor Externo" [en línea]. En: La Plataforma Internet de la Traducción Española en el Parlamento 
Europeo. Luxemburgo: Parlamento Europeo. 1999. [Consulta: 14/01/2010]. Disponible en Internet:

<http://www.europarl.europa.eu/transl_es/plataforma/pagina/maletin/ maletin.htm>

Peláez MARón, J.M. Lecciones de instituciones jurídicas de la Unión Europea. La edición. Madrid: Tecnos. 2000.

PILEGAARD, Morten "Linguistic politeness in intercultural business correspondence". Paper in International Pragmatics Conference. Barcelona. 1990.

PYM, Anthony. "The European Union and its Future Languages. Questions for Language Policies and Translation Theories" [en línea]. En: Anthony Pym website. Tarragona. 1999. [Consulta: 05/07/2010]. Disponible en Internet:

< http://www.tinet.cat/ apym/on-line/translation/acrossEU6.pdf>

SEARLE J.R. "The classification of illocutionary acts". En: Language in Society 5. 1976. Pp. 1-24.

STRANDVIK, Ingemar "Transparencia, gobernanza y traducción ¿ha llegado la hora de un enfoque funcional?" [En línea] Comunicación presentada en el I Congreso Internacional: El español, lengua de traducción. Almagro. 2002. [Consulta: 25/06/2010]. Disponible en Internet:

<http://www.esletra.org/Almagro/html/strandvik_corri_es.htm>

SWALES, John: Genre Analysis. Oxford U. Press. 1991.

Trosborg, A. (ed.). Text Typology and Translation, Amsterdam.-Filadelfia: John Benjamins. 1997.

Trosborg, Anna: An analysis of legal speech acts in English Contract Law. Hermes: Arhus, 1991. Pp. 65-90.

WAGNER, Emma: "Translation in the EU Machinery", Perspectives - Studies in translatology, volumen 9:4. 2001. Pp. 263-270.

BECH, Svend Y MARTínEZ, Jesús M. (2002): Translating for the European Union Institutions, St. Jerome Publishing, Manchester. 2002.

Werther, C./Helmersen, O.: Konflikt eller konsensus. Del: II. Sproglige analyser af agent- og eneforhandlerkontrakter. ARK 49. The Copenhagen Business School. 1989. 